\title{
Representing 3-D cloud radiation effects in two-stream schemes: 1. Longwave considerations and effective cloud edge length
}

Article

Published Version

Schafer, S. A. K., Hogan, R. J., Klinger, C., Chiu, J. C. and Mayer, B. (2016) Representing 3-D cloud radiation effects in two-stream schemes: 1. Longwave considerations and effective cloud edge length. Journal of Geophysical Research: Atmospheres, 121 (14). pp. 8567-8582. ISSN 2169-8996 doi: https://doi.org/10.1002/2016JD024876 Available at https://centaur.reading.ac.uk/66451/

It is advisable to refer to the publisher's version if you intend to cite from the work. See Guidance on citing.

To link to this article DOI: http://dx.doi.org/10.1002/2016JD024876

Publisher: American Geophysical Union

All outputs in CentAUR are protected by Intellectual Property Rights law, including copyright law. Copyright and IPR is retained by the creators or other copyright holders. Terms and conditions for use of this material are defined in the End User Agreement. 


\section{www.reading.ac.uk/centaur}

\section{CentAUR}

Central Archive at the University of Reading

Reading's research outputs online 


\section{Journal of Geophysical Research: Atmospheres}

\section{RESEARCH ARTICLE \\ 10.1002/2016JD024876 \\ This article is a companion to Hogan et al. [2016] doi:10.1002/2016JD024875. \\ Representing 3-D cloud radiation effects in two-stream schemes: 1. Longwave considerations and effective cloud edge length}

Key Points:

- Longwave 3-D cloud radiation effects can be up to $30 \%$

- We develop the longwave part of a new fast 3-D radiation scheme

-We account for radiative smoothing, cloud clustering, in-cloud flux distribution

Correspondence to:

S. A. K. Schäfer,

s.a.k.schafer@pgr.reading.ac.uk

\section{Citation:}

Schäfer, S. A. K., R. J. Hogan, C. Klinger, J. C. Chiu, and B. Mayer (2016), Representing 3-D cloud radiation effects in two-stream schemes: 1. Longwave considerations and effective cloud edge length, J. Geophys. Res. Atmos., 121, 8567-8582, doi:10.1002/2016JD024876.

Received 29 JAN 2016 Accepted 9 JUN 2016

Accepted article online 27 JUN 2016 Published online 27 JUL 2016

(O2016. American Geophysical Union All Rights Reserved.

\author{
Sophia A. K. Schäfer ${ }^{1}$, Robin J. Hogan ${ }^{1,2}$, Carolin Klinger $^{3}$, J. Christine Chiu ${ }^{1}$, and Bernhard Mayer ${ }^{3}$ \\ ${ }^{1}$ Department of Meteorology, University of Reading, Reading, UK, ${ }^{2} \mathrm{ECMWF}$, Reading, UK, ${ }^{3}$ Meteorological Institute, \\ Ludwig-Maximilians-University, Munich, Germany
}

\begin{abstract}
Current weather and climate models neglect 3-D radiative transfer through cloud sides, which can change the cloud radiative effect (CRE) significantly. This two-part paper describes the development of the SPeedy Algorithm for Radiative TrAnsfer through CloUd Sides (SPARTACUS) to capture these effects efficiently in a two-stream radiation scheme for use in global models. The present paper concerns the longwave spectral region, where not much work has been done previously, although the limited previous work has suggested that radiative transfer through cloud sides increases the longwave surface CRE of shallow cumulus by around $30 \%$. To assist the development of a longwave capability for SPARTACUS, we use a reference case of an isolated, isothermal, optically thick, cubic cloud in vacuum, for which 3-D effects increase CRE by exactly $200 \%$. It is shown that for any cloud shape, the 3-D effect can be represented in SPARTACUS provided that correct account is made for (1) the effective zenith angle of diffuse radiation emitted from a cloud, (2) the spatial distribution of fluxes in the cloud, (3) cloud clustering that enhances the interception of emitted radiation by neighboring clouds, and (4) radiative smoothing leading to the effective cloud edge length being less than the measured value. We find empirically that the circumference of an ellipse fitted to a horizontal cross section through a cumulus cloud provides a good estimate of the radiatively effective cloud edge length, which provides some guidance to how cloud observations could be analyzed to extract their most important properties for radiation.
\end{abstract}

\section{Introduction}

Clouds are a key component of the climate system: they strongly influence the radiative gain and loss of energy that drives atmospheric processes on all scales, from global temperature patterns to microphysical processes [Boucher et al., 2013]. Their complex 3-D shapes and high spatial and temporal variability make their representation in models challenging. Numerical radiative transfer schemes in weather and climate models have traditionally relied on several assumptions to simplify the problem, such as that the cloudy region of a model grid box can be treated as horizontally homogeneous, neglecting the horizontal variability observed in real clouds (the plane-parallel assumption). The development of fast radiation schemes able to treat cloud horizontal structure has now largely solved this problem [e.g., Pincus et al., 2003; Shonk and Hogan, 2008], and they have been used to estimate that the plane-parallel approximation results in an overestimation of the magnitude of the net longwave plus shortwave cloud radiative effect (CRE) at top of atmosphere (TOA) by around 14\% globally [Shonk and Hogan, 2010].

A further assumption of such models is that it is sufficient to treat vertical radiative fluxes within cloudy and clear regions separately, without including horizontal transport between them (the 1-D or independent column approximation, abbreviated ICA). In the shortwave spectral region, this assumption induces an error in CRE at TOA of between $-25 \%$ and $+100 \%$ (depending on solar zenith angle) in individual scenes of strongly non-plane-parallel clouds such as cumulus, contrails, or deep convection [Benner and Evans, 2001; DiGiuseppe and Tompkins, 2003; Gounou and Hogan, 2007] but considerably less for stratocumulus and cirrus [Zuidema and Evans, 1998; Zhong et al., 2008]. For longwave radiation, much less work has been done, and longwave 3-D effects are often assumed to be negligible. However, studies have estimated that 3-D effects increase longwave surface CRE by $30 \%$ for cumulus [Heidinger and Cox, 1996] and both TOA and surface longwave CRE by $10 \%$ for aircraft contrails [Gounou and Hogan, 2007]. Takara and Ellingson [2000] have reported surface longwave flux errors of up to $10 \mathrm{Wm}^{-2}$ due to neglecting both 3-D effects and longwave scattering. It should be 
noted that 3-D longwave effects are largest at the surface, because their strongest contributions come from optically thick broken clouds like cumulus that occur low in the atmosphere. At TOA on the other hand, the global study of Cole et al. [2005], using a 2-D cloud model nested in the global model, found a smaller increase in longwave CRE of less than $1 \mathrm{Wm}^{-2}$ in zonal averages. We expect the observed 3-D effect to be lower in studies that use 2-D approximations (which neglect one dimension of horizontal radiative transfer) or at coarse resolutions like the $4 \mathrm{~km}$ used in Cole et al. [2005] (which neglect some of the cloud structure).

While high-resolution 3-D radiation models are capable of calculating the precise radiative effect of a known 3-D cloud field [e.g., Cahalan et al., 2005; Pincus and Evans, 2009; Mayer, 2009], they are not suitable for climate models because of their high computation cost. Killen and Ellingson [1994] and Heidinger and Cox [1996] proposed simple scaling of the cloud fraction as an empirical approximation to account for 3-D effects, but that method is difficult to implement for general cloud fields, especially with multiple cloud layers. Consistency with cloud effects in the shortwave is also hard to achieve, as any equivalent shortwave method would only hold for one particular solar zenith angle. We seek a more sophisticated method that is physically consistent between spectral regions and valid for general cloud fields. Recently, fast approximations for 3-D radiative transfer on cloud-resolving scales have been developed [Klinger and Mayer, 2016; Jakub and Mayer, 2015], but these approaches are not designed for use in global models.

The lack of a fast broadband radiative transfer scheme that can reliably represent 3-D effects in large-scale models means that we currently have no way to estimate the importance of 3-D radiative transfer on the Earth's radiation budget or on the evolution of weather systems. As a first step to tackling this issue, Hogan and Shonk [2013] proposed a method to incorporate 3-D effects into a conventional two-stream radiative transfer code. Their approach describes 3-D cloud structure information in terms of cloud side area (or equivalently, the cloud edge length of a horizontal slice through a cloud field) and adds terms to the two-stream equations to represent the loss and gain of radiation through cloud sides, with a modest increase in computational cost. The assumption underlying this formulation is that the fraction of clear-sky flux that encounters a cloud edge is proportional to the length of cloud edge, which roughly corresponds to assuming a random horizontal distribution of clouds within a grid box. Thus, the cloud edge length versus height, which may be expressed in terms of an effective cloud size [Jensen et al., 2008], is the only input that is needed in addition to the large-scale information on cloud fraction and water content provided in global models. However, Hogan and Shonk [2013] only considered monochromatic calculations in the shortwave part of the spectrum; they neglected in-cloud horizontal inhomogeneity, and their method for solving the modified two-stream equations was excessively complicated, with multiple steps that still resulted in numerical imprecisions and a dependence on vertical resolution.

In this two-part paper we describe the development of a broadband radiation scheme ready for use in large-scale models, which overcomes the limitations of Hogan and Shonk [2013]. We refer to it as the SPeedy Algorithm for Radiative TrAnsfer through CloUd Sides (SPARTACUS). Hogan et al. [2016, hereinafter Part 2] incorporates treatment of cloud inhomogeneity using the Tripleclouds method following Shonk and Hogan [2008] and describes an elegant and accurate method for solving the modified two-stream equations using matrix exponentials. They also evaluate the method against fully 3-D broadband radiative transfer calculations in both the shortwave and longwave.

In this paper (Part 1), we develop the longwave capability of SPARTACUS making use of fully 3-D calculations to identify the most important features to represent, as well as introducing improvements that are applicable in both the shortwave and the longwave. Section 2 describes the idealized case of an isolated, isothermal, cubic cloud, which is a very useful benchmark for longwave 3-D radiation schemes because its known symmetry properties allow us to determine the 3-D effect analytically. In section 3, we outline the SPARTACUS scheme and use the results of section 2 to develop a consistent longwave capability. As part of this we correct inconsistencies in the treatment of horizontal and vertical fluxes by Hogan and Shonk [2013]. Tests using realistic cumulus clouds are performed in section 4, including the two main factors important for determining the radiatively effective cloud edge length: cloud clustering and radiative smoothing. This provides guidance for the use of observations to quantify the cloud geometry variable needed by SPARTACUS when applied to realistic cloud fields. The performance of SPARTACUS is compared to full 3-D simulations with the Monte Carlo code for the physically correct tracing of photons in cloud atmospheres (MYSTIC) [Mayer, 2009]. 


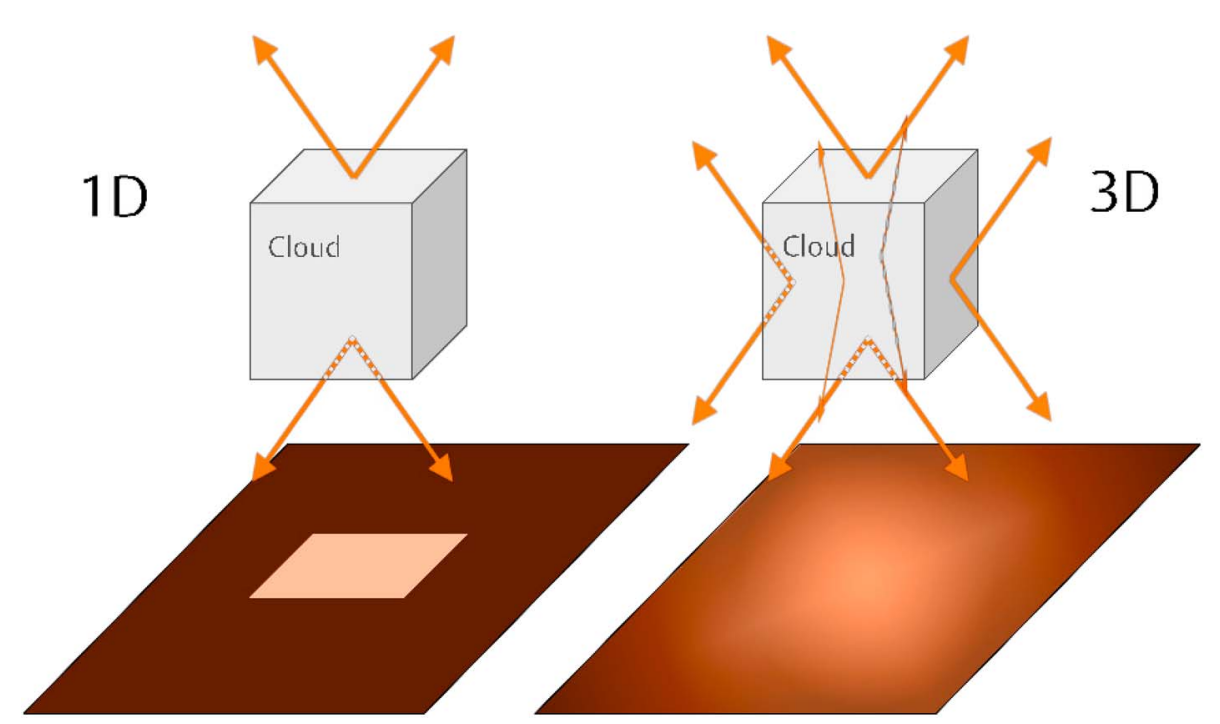

Figure 1. Schematic of outgoing fluxes and their contributions to total downwelling flux in 1-D and 3-D schemes for a cubic cloud. Because of symmetry, the total outward flux $G$ through every cloud face is the same, as discussed in section 2. Every arrow symbolizes a flux of $G / 2$ through the respective face. At cloud sides, half of the outgoing radiation is at an upward angle, the other half at a downward angle. The distribution of downwelling flux at the surface is shown below: in a 1-D scheme, we only see downwelling flux directly underneath the cloud, while in a 3-D scheme, cloud side fluxes result in a more spread-out distribution as well as in higher total downwelling flux.

\section{Theory for an Idealized Cubic Cloud}

In this section, we examine the idealized case of one homogeneous, isothermal, cubic cloud in vacuum that is isolated in a larger model grid box of surface area $A$ without other clouds (so cloud fraction $c$ is small). In this symmetric case, the 3-D effects can be derived analytically, providing an excellent benchmark for 3-D radiation schemes (both fully 3-D schemes and approximate schemes such as SPARTACUS). As we are interested in the cloud longwave effect, we here consider only thermal radiation emitted from the cloud, without direct solar radiation or emission, reflection or absorption by the ground. More realistic cases including atmospheric and surface emission, absorption, and reflection will be considered in Part 2.

All properties of a homogeneous, isothermal cubic cloud are symmetric with respect to discrete rotations that exchange the faces of the cube, and therefore so is the radiation emitted at each face. This means that the outward flux through each cloud face is the same, $G$, shown schematically in Figure 1. Here "outward flux" is defined as the radiation through a plane parallel to the cloud face, per unit area of the face (we denote fluxes per area of the cloud by $G$ and fluxes per horizontal area of the domain by $F$ ).

We can derive the theoretical cloud 3-D effect by comparing the different contributions to the total downward flux $F^{\downarrow}$ through a horizontal plane, per unit area of the entire grid box (note difference in definition of flux). The fraction of $G$ through a cloud face that contributes to downward flux depends on the orientation of the face. At cloud base, outward is the same as downward, so $F_{\text {base }}^{\downarrow}=c G$, where the cloud fraction $c=A_{\text {base }} / A$ accounts for the difference between flux per area of cloud base $A_{\text {base }}$ and flux per total grid box area $A$. At a cloud side, on the other hand, radiation can leave the cloud at an upward or a downward angle with equal probability. Therefore, half of the radiation from each cloud side contributes to the downward flux: $F_{\text {side }}^{\downarrow}=\frac{1}{2} G A_{\text {side }} / A$, and since $A_{\text {side }}=A_{\text {base, }}$, this becomes $F_{\text {side }}^{\downarrow}=c G / 2$.

Adding the fluxes from cloud base and the four cloud sides gives

$$
F_{\text {base+sides }}^{\downarrow}=F_{\text {base }}^{\downarrow}+4 F_{\text {side }}^{\downarrow}=3 c G \text {. }
$$

As a measure of the 3-D cloud side effect, we introduce the cloud side factor $f_{\text {sides }}=F_{\text {base+sides }}^{\downarrow} / F_{\text {base }}^{\downarrow}$. If the cloud is in a vacuum and there is no interaction with the ground, then from (1) we see that $f_{\text {sides }}=3$ at all heights beneath the cloud.

This result gives an idea of the importance of 3-D effects: two thirds of the downwelling radiation from the cloud comes from the cloud sides, not the cloud base. The theory is independent of the optical depth of the cloud or the particle scattering properties, allowing the following general statement: 


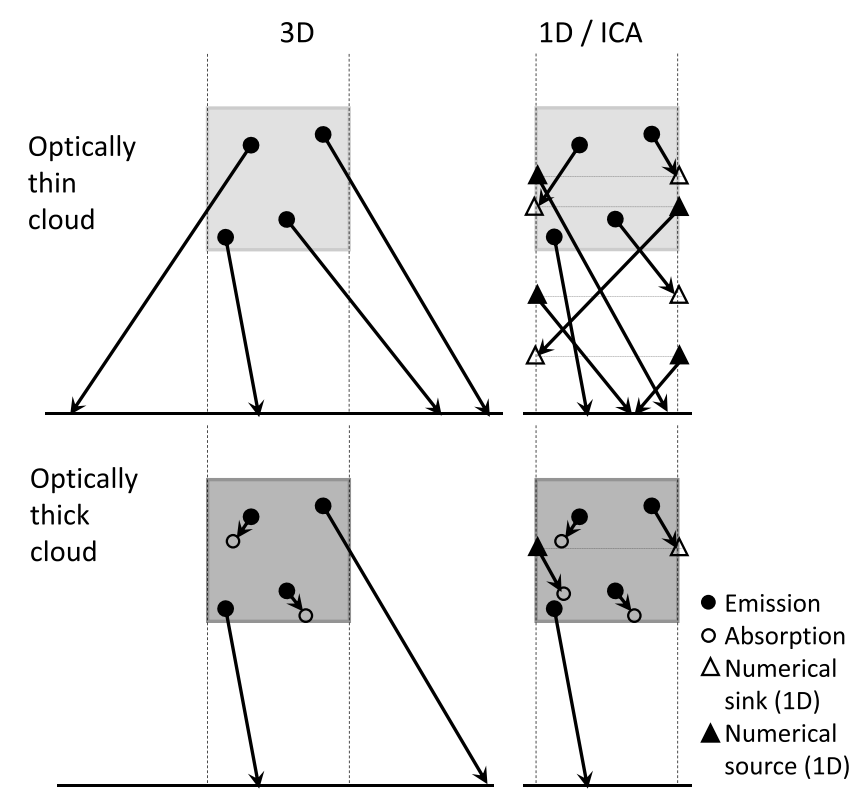

Figure 2. Schematic showing the treatment of downwelling radiation encountering cloud sides in 3-D and 1-D schemes, in the limit of (top row) optically thin and (bottom row) optically thick clouds, for fully 3-D radiative transfer (in the left column) and the 1-D independent column approximation (ICA; in the right column). In the ICA, cloudy columns have periodic boundary conditions, so that radiation that encounters the side of the column reenters on the opposite side (shown as numerical sinks and sources in the plot). This makes the radiation's path through the cloud longer than in reality. In the optically thick case increased absorption along the longer ICA path is noticeable, which leads to an underestimation of downwelling flux below the cloud.

Theorem 1. For an isolated, isothermal, homogeneous cubic cloud in vacuum above a nonreflecting, nonemitting surface, a third of the downwelling radiation beneath the cloud originates from the cloud base and two thirds from the cloud sides, regardless of the optical depth or scattering properties of the cloud. The same holds for upwelling emitted radiation above the cloud: one third originates from cloud top and two thirds from cloud sides.

In realistic cases with gaseous absorption and emission, the cloud side factor could be defined as the ratio of cloud radiative effect resulting from outgoing fluxes through all faces to that from outgoing fluxes through cloud top and base alone. In these cases, the factor is likely to be somewhat less, partly due to increased gaseous absorption on the longer slanted paths to the ground for radiation from cloud sides, but as we will show, the 3-D effect should not be neglected and always leads to an increase in cloud radiative effect, in contrast to the shortwave case, where it can be of either sign.

While the cloud side factor measures the importance of cloud side emission, we are particularly interested in the change in cloud radiative effect relative to the results of current 1-D schemes of the type used in weather and climate models. Figure 2 shows how radiation encountering cloud sides is treated in 3-D and 1-D schemes. In 1-D schemes, periodic boundary conditions at region boundaries let radiation that encounters a cloud side reenter at the opposite side and remain within the cloudy region (this is shown schematically in Figure 2 as a numerical sink and a numerical source that lets the radiation reenter the cloud at the opposite side).

For very optically thin clouds (optical depth $\tau \ll 1$ ), where the chance of absorption or backscattering (scattering from the downward to the upward direction or vice versa) is small, radiation once emitted is essentially unaffected by its passage through the cloud. Total downwelling flux in the grid box at any height only depends on the number of emitting cloud particles above. Hence, as using 1-D periodic cloud boundary conditions does not change particle emission, the total downwelling flux in the grid box beneath the cloud as predicted by a 1-D scheme $F_{1 \mathrm{D}}^{\downarrow}$ approaches the correct 3-D value $F_{\text {base }+ \text { sides. }}^{\downarrow}$. The spatial distribution of fluxes, however, is different: in reality some radiation leaves the cloudy column and reaches the surface at some horizontal distance from the cloud but still contributes to the grid box total of downwelling flux from the cloud. In the $1-D$ calculation this radiation is instead added to the downward flux directly underneath the cloud, which is thereby increased above its real value (as illustrated in Figure 1). 
For very optically thick clouds $(\tau \gg 1)$, the picture is different. Again, radiation that in reality would escape through cloud sides remains within the cloud in the 1-D scheme, but because of the high optical depth virtually all of it is absorbed or scattered rather than reaching cloud base (see Figure 2). The downwelling flux beneath the cloud $F_{1 \mathrm{D}}^{\downarrow}$ is approximately equal to the flux out of cloud base in the 3-D case $F_{\text {base }}^{\downarrow}$, which, as stated above, is lower by a factor of 3 than the correct total downwelling flux. This gives us a limiting benchmark for the underestimation of cloud radiative effects by 1-D schemes:

Theorem 2. For an isolated, isothermal, homogeneous and very optically thick cubic cloud in vacuum above a nonreflecting, nonemitting surface, the downwelling radiation beneath the cloud and upwelling radiation above the cloud are underestimated by a factor of 3 in 1-D radiation schemes.

The factor $f_{1 \text { Dcorrection }}=F_{3 \mathrm{D}}^{\downarrow} / F_{1 \mathrm{D}}^{\downarrow}$ quantifies this error for different cloud cases, but note that $f_{1 \mathrm{Dcorrection}}$ is only equal to $f_{\text {sides }}$ for very optically thick clouds.

\section{Development of a Longwave Capability for SPARTACUS}

\subsection{Overview of SPARTACUS}

The SPARTACUS scheme modifies the two-stream equations to incorporate horizontal radiative transfer effects by coupling the equations for neighboring clear and cloudy regions (denoted $a$ and $b$, respectively) through the addition of horizontal transfer gain and loss terms. The cloudy region may optionally be divided into two (denoted $b$ and $c$ ) to represent cloud horizontal inhomogeneity following the approach of Shonk and Hogan [2008]. The two-region case (clear and cloudy) results in the following equations for upwelling and downwelling diffuse fluxes $F^{\uparrow}, F^{\downarrow}$ in any individual longwave or shortwave spectral band:

$$
\begin{aligned}
\frac{\mathrm{d} F_{a}^{\downarrow}}{\mathrm{d} z} & =\beta_{a}\left(-\gamma_{1, a} F_{a}^{\downarrow}+\gamma_{2, a} F_{a}^{\uparrow}+S_{a}^{\downarrow}\right)-f_{a b} \varepsilon_{a} F_{a}^{\downarrow}+f_{b a} \varepsilon_{b} F_{b}^{\downarrow} ; \\
-\frac{\mathrm{d} F_{a}^{\uparrow}}{\mathrm{d} z} & =\beta_{a}\left(-\gamma_{1, a} F_{a}^{\uparrow}+\gamma_{2, a} F_{a}^{\downarrow}+S_{a}^{\uparrow}\right)-f_{a b} \varepsilon_{a} F_{a}^{\uparrow}+f_{b a} \varepsilon_{b} F_{b}^{\uparrow} ; \\
\frac{\mathrm{d} F_{b}^{\downarrow}}{\mathrm{d} z} & =\beta_{b}\left(-\gamma_{1, b} F_{b}^{\downarrow}+\gamma_{2, b} F_{b}^{\uparrow}+S_{b}^{\downarrow}\right)-f_{b a} \varepsilon_{b} F_{b}^{\downarrow}+f_{a b} \varepsilon_{a} F_{a}^{\downarrow} ; \\
-\frac{\mathrm{d} F_{b}^{\uparrow}}{\mathrm{d} z} & =\beta_{b}\left(-\gamma_{1, b} F_{b}^{\uparrow}+\gamma_{2, b} F_{b}^{\downarrow}+S_{b}^{\uparrow}\right)-f_{b a} \varepsilon_{b} F_{b}^{\uparrow}+f_{a b} \varepsilon_{a} F_{a}^{\uparrow},
\end{aligned}
$$

which is analogous to the formalism for diffuse shortwave fluxes in Hogan and Shonk's [2013] equation (11) except for the factors $\varepsilon$ that describe spatial distribution of emitted radiation within each region (see below).

Here the fluxes are radiative power per area of the entire grid box (in $\mathrm{W} \mathrm{m}^{-2}$ ), $z$ is height increasing downward, and $\beta$ is the volume extinction coefficient. The coefficients $\gamma_{1}$ and $\gamma_{2}$ govern extinction by absorption and backscattering, and gain by backscattering, respectively, and are given in equations (12) and (13) of Hogan and Shonk [2013] and elsewhere. The source terms $S^{\downarrow \uparrow}$ are the internal sources of diffuse radiation in each region. In the shortwave this would be scattering from the direct solar beam, while in the longwave it represents isotropic thermal emission. For region $a$ they are given by

$$
S_{a}^{\downarrow}(z)=S_{a}^{\uparrow}(z)=c_{a} \frac{\pi\left(1-\omega_{a}\right)}{\cos \theta_{1}} B[T(z)],
$$

where $c_{a}$ is the fraction of the domain covered by region $a, B$ is the Planck function, $T(z)$ is the temperature at a given height, and $\omega_{a}$ is the single scattering albedo (and similarly for regions $b$ and $c$ ). For optimum longwave results in atmospheres dominated by gas absorption, we assume the zenith angle of diffuse streams, $\theta_{1}$, to be $53^{\circ}$ [Elsasser, 1942; Fu et al., 1997], although other assumptions are possible. Note that $\theta_{1}$ also appears in the definitions of $\gamma_{1}$ and $\gamma_{2}$.

The system of differential equations in (2) can be solved for the entire multilayer atmosphere by formulating the terms as vectors and matrices and computing the solution in terms of matrix exponentials, as described in detail in sections 2 and 3 of Part 2. This approach is more elegant and accurate than the multistage method proposed by Hogan and Shonk [2013]. 
The final two terms on the right-hand side of each of the expressions in (2) represent lateral transport between regions. For example, $f_{b a} \varepsilon_{b} F_{b}^{\downarrow}$ is the rate of transport of downwelling radiation from region $b$ (cloud) to region $a$ (clear sky), per unit vertical distance. Naturally, this term is proportional to $F_{b}^{\downarrow}$, the horizontal-mean flux at a particular height within the cloud. The two coefficients describe the distinct physical processes that determine this rate.

The first coefficient, $f_{a b}$ or $f_{b a}$, is purely a function of the geometry of the cloud edge. Hogan and Shonk [2013] showed that it may be formulated at a given height in terms of the total length of cloud edge per unit area of the grid box. However, applying their theory to the idealized cubic cloud described in section 2 reveals an error, which we address in section 3.2. The second coefficient, $\varepsilon_{b}$, accounts for any systematic difference between the mean fluxes in the cloudy region and fluxes near cloud edge (which determine outgoing fluxes at cloud edge). We choose the symbol $\varepsilon$ as this coefficient plays the role of a kind of effective emissivity of the cloud edge. In the shortwave, we find that $\varepsilon_{b} \simeq 1$ (as assumed by Hogan and Shonk [2013]) works reasonably well, which will be demonstrated in Part 2 from the good agreement found with fully 3-D shortwave calculations. This suggests that there is no systematic increase or decrease of in-cloud shortwave fluxes toward cloud edge. In the longwave, however, emission from within the cloud makes fluxes spatially more variable and $\varepsilon_{b}$ is significantly different from 1 . This effect is parameterized in section 3.3.

While the distribution of fluxes in the clear sky also varies, the variance mostly depends on the distance from clouds, as these dominate the emission. We find it satisfactory to include this effect in the parameterization of cloud geometry (described in section 4 ) and hence set $\varepsilon_{a}$ to 1 .

\subsection{Effective Direction of Radiation Transported Through Cloud Sides}

This section derives an expression for the coefficients $f_{a b}$ and $f_{b a}$ in (2), which describe the contribution to the rate of lateral exchange from the geometry of the cloud edge. Hogan and Shonk [2013] showed that if the diffuse radiation is assumed to be traveling in discrete directions with zenith angles of $\theta_{3 \mathrm{D}}$ and $\pi-\theta_{3 \mathrm{D}}$ (illustrated in Figure 3a), then the coefficients are given by

$$
f_{a b}=\tan \left(\theta_{3 \mathrm{D}}\right) \frac{L_{a b}}{\pi c_{a}} ; \quad f_{b a}=\tan \left(\theta_{3 \mathrm{D}}\right) \frac{L_{a b}}{\pi c_{b}},
$$

where $c_{a}$ and $c_{b}$ are the fractions of the domain covered by regions $a$ and $b$, respectively, and $L_{a b}$ is the length of cloud edge (i.e., the length of the interface between regions $a$ and $b$ in the horizontal plane) per unit area of the grid box.

Hogan and Shonk [2013] equated $\theta_{3 \mathrm{D}}$ with the diffusivity angle $\theta_{1}$ used in the definitions of $\gamma_{1}, \gamma_{2}$, and $S^{\downarrow \uparrow}$ and hence used $\theta_{3 \mathrm{D}}=53^{\circ}$. To test this, we appeal to the idealized case described in section 2 and use SPARTACUS to simulate emission from an isothermal, cubic cloud in vacuum. In order to isolate purely the geometric effect, we consider a very optically thick nonscattering cloud so that the effective emissivity of the cloud, $\varepsilon_{b}$, is unity. The dash-dotted black line in Figure $3 \mathrm{~b}$ depicts the 3-D factor $f_{1 \text { Dcorrection }}$ as a function of cloud fraction for $\theta_{3 \mathrm{D}}=53^{\circ}$, and it can be seen that the 3-D effect reduces for increasing cloud fraction due to the increased probability of radiation emitted from the side of a cloud being absorbed by another cloud. In the limit of completely overcast skies, SPARTACUS has the correct limit of no 3-D effect. However, in the limit of very small cloud fraction, $f_{1 \text { Dcorrection }}$ has a value of 2.65 rather than the value of exactly 3 both predicted by Theorem 2 in section 2 and simulated by the fully 3-D Monte Carlo radiation model MYSTIC [Mayer, 2009]. This means that the isothermal cube is incorrectly emitting less from its sides than from its base in SPARTACUS.

MYSTIC, which is used here and throughout this paper to evaluate SPARTACUS, has been validated by extensive comparison with other 3-D state-of-the-art radiative transfer models [Cahalan et al., 2005] and both shortwave and longwave observations [Mayer et al., 2010; Emde and Mayer, 2007] and showed very good agreement. Klinger and Mayer [2014] showed that completely independent approaches in MYSTIC for calculating thermal heating and cooling rates are consistent with each other. For this paper, thermal fluxes were calculated using $10^{5}$ photons which resulted in a statistical noise of less than $0.1 \%$ for domain-averaged fluxes as shown here.

The red lines in Figure 3b show the results for a cubic cloud with an optical depth of 2; it can be seen that $f_{1 \text { Dcorrection }}$ is reduced from the value of 3 that is applicable only in the optically thick limit. The two red lines also confirm that SPARTACUS is not sensitive to vertical resolution. The details of how SPARTACUS is run with multiple layers are given in Part 2. 
(a)

(b)
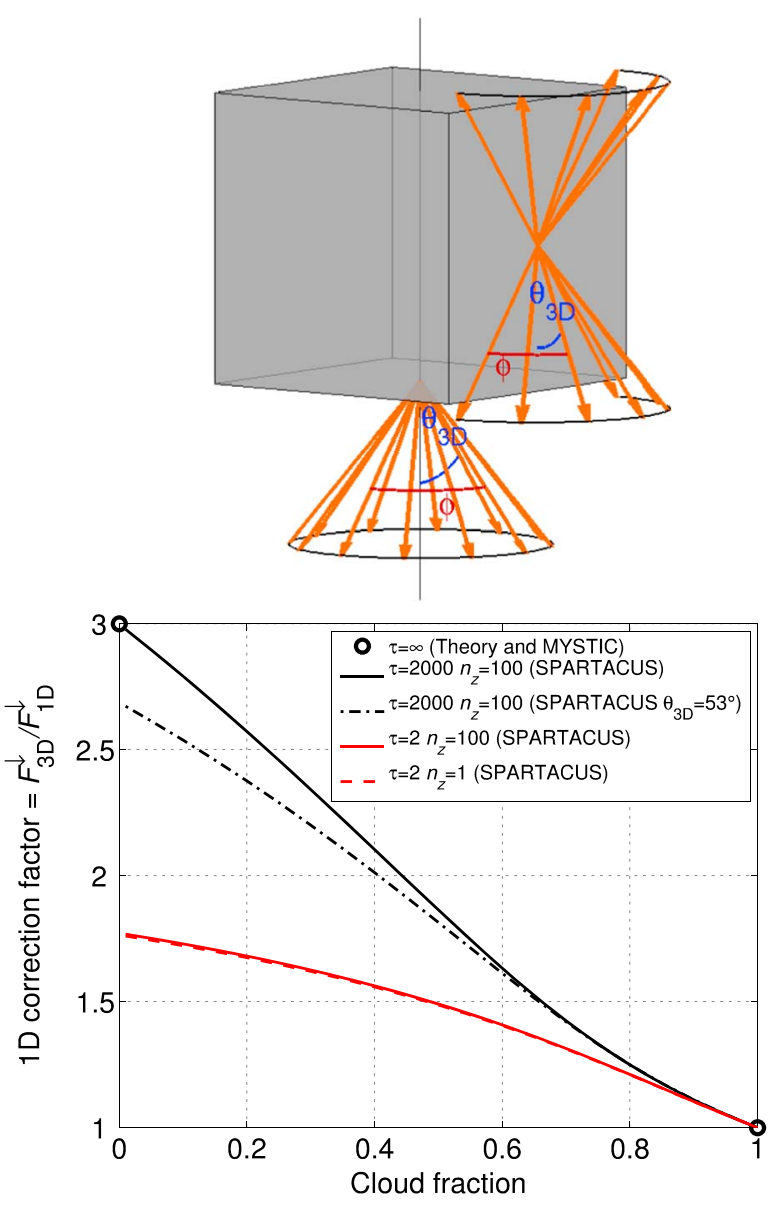

Figure 3. (a) Schematic of outward radiances from cloud base and cloud side in the approximation of discrete zenith angles $\theta_{3 \mathrm{D}}$ and $\pi-\theta_{3 \mathrm{D}}$ for a cubic cloud. (b) Dependence of 3-D factor, denoted $f_{1 \mathrm{Dcorrection}}$ in section 2, on cloud fraction, for an isothermal, cubic cloud in vacuum, where optical depth is denoted in the legend by $\tau$ and the number of vertical layers by $n_{z}$. All SPARTACUS simulations use the effective zenith angle for lateral transport derived in section 3.2 of $\theta_{3 \mathrm{D}}=57.52^{\circ}$, except for the black dash-dotted line that uses a value of $53^{\circ}$. The circles show the theoretical limits of very low cloud fraction and overcast sky for a very optically thick cloud, which agree with MYSTIC calculations.

We wish to derive new expressions for $f_{a b}$ and $f_{b a}$ that ensure that vertical transport and horizontal transport between clear and cloudy regions are treated consistently. This can be thought of as finding a better value for $\theta_{3 \mathrm{D}}$, but we need not make the assumption that diffuse radiation travels only with two discrete zenith angles. We again consider a very optically thick nonscattering cloud, which can now have any shape. In this case, it can be seen from (2) that the rate of change of in-cloud fluxes due to lateral escape through the cloud sides is given by

$$
\left.\frac{\mathrm{d} F_{b}^{\downarrow}}{\mathrm{d} z}\right|_{\text {lat }}=-f_{b a} F_{b}^{\downarrow} ; \quad-\left.\frac{\mathrm{d} F_{b}^{\uparrow}}{\mathrm{d} z}\right|_{\text {lat }}=-f_{b a} F_{b}^{\uparrow} .
$$

The flux exiting the cloud sides over a thin layer of depth $\mathrm{d} z$, per area of the entire grid box, is then

$$
-\mathrm{d} F_{b}^{\downarrow}+\mathrm{d} F_{b}^{\uparrow}=f_{b a}\left(F_{b}^{\downarrow}+F_{b}^{\uparrow}\right) \mathrm{d} z
$$

From this, we can compute the flux exiting a thin layer of the cloud side per unit area of cloud side, $G_{\text {side, }}$ by dividing the left-hand side of (5) by the ratio of cloud side area in the thin layer to the horizontal area of the grid box, $L_{a b} \mathrm{~d} z$, yielding: $G_{\text {side }}=f_{b a}\left(F_{b}^{\downarrow}+F_{b}^{\uparrow}\right) / L_{a b}$. Since upwelling and downwelling radiation within the optically thick cloud are in equilibrium (as explained in section 3.3.2) is isotropic, we have $F_{b}^{\downarrow}=F_{b}^{\uparrow}$ and hence

$$
G_{\text {side }}=2 f_{b a} F_{b}^{\downarrow} / L_{a b}
$$


Meanwhile, the flux exiting the cloud base per unit area of cloud base is simply the downwelling flux $F_{b}^{\downarrow}$ (which is per unit area of the grid box) divided by the cloud fraction:

$$
G_{\text {base }}=F_{b}^{\downarrow} / c_{b} \text {. }
$$

We require that the outgoing diffuse fluxes out of the base and sides are the same, i.e., $G_{\text {base }}=G_{\text {side, }}$ so equating (6) and (7) yields

$$
f_{b a}=\frac{L_{a b}}{2 c_{b}}
$$

This may be used as a direct replacement for (3).

While the derivation of (8) has not assumed that the diffuse radiation travels in discrete directions, it is illuminating to equate (3) and (8), which reveals that the effective zenith angle to get the correct lateral transport is $\theta_{3 \mathrm{D}}=57.52^{\circ}$. The fact that this value is larger than $53^{\circ}$ means that it will lead to a larger 3-D effect than in Hogan and Shonk [2013], and indeed Figure $3 \mathrm{~b}$ shows that when the new value is used, SPARTACUS yields the correct 3-D effect (a value of $f_{1 \text { Dcorrection }}=3$ ) for the isolated optically thick cubic cloud considered in section 2 .

As an alternative way to derive this angle, consider the approximation of all diffuse radiation being represented as upward and downward cones at zenith angles $\theta_{3 \mathrm{D}}$ and $\pi-\theta_{3 \mathrm{D}}$, respectively, both with radiance $I$, as illustrated in Figure 3a. As each cloud particle's emission is isotropic, it is reasonable to assume that if the cloud's height and width are similar (as fulfilled for a typical cumulus cloud), the downward flux per area of horizontal cloud surface should equal the lateral flux per area of vertical cloud surface. The downward flux is found by integrating over all azimuth angles $\phi$ of the downward cone:

$$
G_{\text {base }}=\int_{0}^{2 \pi} I \cos \theta_{3 \mathrm{D}} \mathrm{d} \phi=2 \pi l \cos \theta_{3 \mathrm{D}}
$$

If $\phi$ represents the azimuth angle of a beam of radiation with respect to the normal to a vertical surface representing part of the cloud side, then the flux through that surface is

$$
G_{\text {side }}=2 \int_{-\pi / 2}^{\pi / 2} I \sin \theta_{3 \mathrm{D}} \cos \phi \mathrm{d} \phi=4 / \sin \theta_{3 \mathrm{D}} .
$$

Since emission per surface area of cloud base and cloud side should be the same, we set $G_{\text {base }}=G_{\text {side, }}$, which also yields $\theta_{3 \mathrm{D}}=57.52^{\circ}$.

\subsection{Horizontal Distribution of Fluxes in Cloud}

In reality, the rate at which radiation escapes through the side of a cloud is proportional to the local value of the upwelling and downwelling diffuse flux in the cloud very near the cloud edge. This section parameterizes the coefficient $\varepsilon_{b}$ in (2), which quantifies the ratio of the near-edge fluxes to the mean flux in the cloud. Hogan and Shonk [2013] assumed fluxes to be homogeneous within the cloud, so that $\varepsilon_{b}=1$. We can only be sure that this is valid in the longwave for optically thick nonscattering clouds (as assumed in section 3.2).

To test the validity of assuming $\varepsilon_{b}=1$ over a wider range of cloud properties, we again appeal to Theorem 1 in section 2, which states that for an isothermal, homogeneous, isolated cubic cloud in vacuum, the outgoing lateral flux from each side face $G_{\text {side }}$ equals the flux from top or base, $G_{\text {base }}$. Fully 3-D calculations using MYSTIC have confirmed that the ratio $G_{\text {side }} / G_{\text {base }}=1$ for a range of scattering properties. We have computed this ratio with SPARTACUS assuming $\varepsilon_{b}=1$ for various optical depths and single scattering albedos $\omega$, as is shown by the dashed lines in Figure 4 . It is clear that $\varepsilon_{b}=1$ performs poorly in general: in optically thin clouds the side flux is underestimated for all values of $\omega$, because in reality lateral fluxes due to internal emission accumulate toward the edges of the cloud, making cloud edge fluxes just before escape larger than the mean in-cloud values. For optically thick clouds the side flux is overestimated, but only for strongly scattering clouds; this is because larger $\omega$ reduces the emissivity of the cloud edge, an effect not captured by SPARTACUS.

It should be stressed that these two phenomena also affect the outgoing fluxes at cloud top and base. The difference is that there they are fully represented in SPARTACUS via its use of the classical two-stream equations including explicit representation of the vertical exchange of radiation between upwelling and downwelling streams. Since SPARTACUS does not similarly resolve horizontally oriented fluxes and the exchange between 


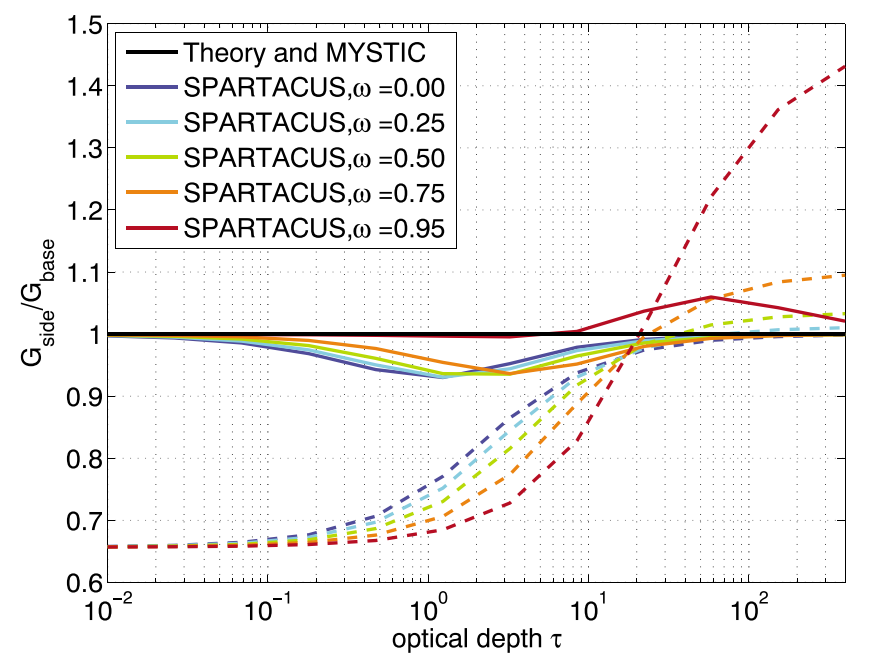

Figure 4. Ratio of horizontal to vertical outgoing fluxes $G_{\text {side }} / G_{\text {base }}$ from an isothermal homogeneous cubic cloud in vacuum at wavelength $\lambda=10.7 \mu \mathrm{m}$ (asymmetry factor $g=0.85$ ) in SPARTACUS assuming $\varepsilon_{b}=1$ (dashed lines) and with a full parameterization of $\varepsilon_{b}$ (solid lines), versus the logarithm of optical depth $\tau$ for different values of single scattering albedo $\omega$. Both theory and fully 3-D calculations using MYSTIC predict a value of unity for this ratio, shown by the black horizontal line.

radiation traveling toward and away from a cloud edge, these two phenomena are not simulated automatically, so they must be parameterized via the specification of $\varepsilon_{b}$ in order to obtain consistent behavior at cloud sides as at cloud base and top.

Our approach to parameterizing $\varepsilon_{b}$ is to use the two-stream equations to describe how outgoing horizontal fluxes at cloud sides relate to the cloud average fluxes, depending on the cloud's optical properties. In the limiting cases of very optically thin or very optically thick clouds, the equations simplify enough to be solved analytically (sections 3.3.1 and 3.3.2, respectively), and in section 3.3.3 we combine these findings to obtain a parameterization that may be used over the full range of optical depth.

\subsubsection{Optically Thin Limit}

As the two-stream scheme in SPARTACUS already calculates fluxes out of vertical surfaces correctly, we can ask the question what value of $\varepsilon_{b}$ results in the same lateral as vertical outgoing cloud edge fluxes, for the case of the isothermal homogeneous cubic cloud in vacuum. We denote the value in the limit of very low optical depth as $\varepsilon_{0, b}$. The fact that the bias at low optical depth shown in Figure 4 is insensitive to $\omega$ suggests that $\varepsilon_{0, b}$ will be independent of $\omega$. A limitation of this section is that our use of a cubic cloud means that strictly the result will only be applicable for clouds with an aspect ratio of around unity, although we note that 3-D effects get weaker as optical depth decreases, so this is not expected to have a strong impact on any future estimate of the global impact of 3-D radiative effects.

In the optically thin limit, radiation emitted from one cloud particle is very unlikely to encounter another before it leaves the cloud, so we can neglect the scattering and absorption terms in (2) and the upwelling and downwelling streams decouple. Thus, the equation for the downwelling in-cloud flux becomes

$$
\frac{\mathrm{d} F_{b}^{\downarrow}}{\mathrm{d} z}=\beta_{b} S_{b}^{\downarrow}-f_{b a} \varepsilon_{0, b} F_{b}^{\downarrow},
$$

which has a general solution

$$
F_{b}^{\downarrow}(z)=X \exp \left(-f_{b a} \varepsilon_{0, b} z\right)+\frac{\beta_{b} S_{b}^{\downarrow}}{f_{b a} \varepsilon_{0, b}},
$$

where $X$ is a real number. Since we are concerned with radiation originating from within the cloud, we insert the boundary condition of no downward flux at cloud top, i.e., $F_{b}^{\downarrow}(z=0)=0$, which gives $X=-\beta_{b} S_{b}^{\downarrow} / f_{b a} \varepsilon_{0, b}$, and therefore

$$
F_{b}^{\downarrow}(z)=\frac{\beta_{b} S_{b}^{\downarrow}}{f_{b a} \varepsilon_{0, b}}\left[1-\exp \left(-f_{b a} \varepsilon_{0, b} z\right)\right]
$$


Similarly, the equation for downwelling clear-sky flux due to emission from cloud sides (i.e., without internal clear-sky sources) reduces to

$$
\frac{\mathrm{d} F_{a}^{\downarrow}}{\mathrm{d} z}=f_{b a} \varepsilon_{0, b} F_{b}^{\downarrow}=\beta_{b} S_{b}^{\downarrow}\left[1-\exp \left(-f_{b a} \varepsilon_{0, b} z\right)\right]
$$

The solution, again using the zero upper boundary condition $F_{a}^{\downarrow}(z=0)=0$, is

$$
F_{a}^{\downarrow}(z)=\beta_{b} S_{b}^{\downarrow}\left[z+\frac{\exp \left(-f_{b a} \varepsilon_{0, b} z\right)-1}{f_{b a} \varepsilon_{0, b}}\right] .
$$

We consider a cloud with a vertical depth of $\Delta z$; hence, at the level of cloud base, $F_{b}^{\downarrow}(\Delta z)$ is the radiation emerging from cloud base and $F_{a}^{\downarrow}(\Delta z)$ is the radiation that originated from the cloud sides. From Theorem 1 in section 2, we know for a cubic, isothermal cloud that

$$
F_{a}^{\downarrow}(\Delta z)=2 F_{b}^{\downarrow}(\Delta z)
$$

Substituting in (12) and (14) and simplifying yields the following implicit equation for $\varepsilon_{0, b}$ :

$$
3 \exp \left(-\varepsilon_{0, b} f_{b a} \Delta z\right)+\varepsilon_{0, b} f_{b a} \Delta z=3
$$

For a cubic cloud with depth $\Delta z$ in a grid box of area $A$, the cloud fraction is $c_{b}=\Delta z^{2} / A$ and the cloud edge length per unit area is $L_{a b}=4 \Delta z / A$. Combining with the definition of $f_{b a}$ in (8) indicates that $f_{b a} \Delta z=2$. The numerical solution in this case is $\varepsilon_{0, b}=1.4107$. (Equation (16) for $f_{b a} \Delta z=2$ actually has an analytical solution: $\varepsilon_{0, b}=\left[W\left(-3 e^{-3}\right)+3\right] / 2=1.4107$, where $W$ is the Lambert $W$ function.) As expected, $\varepsilon_{0, b}>1$, to represent the accumulation of outward emission toward the cloud edge that results in a higher flux than the in-cloud average.

\subsubsection{Optically Thick Limit}

In the interior of a very optically thick cloud, the radiance in any direction is equal to the Planck function at the temperature of the cloud, but if the cloud particles have a nonzero single scattering albedo, then the flux emitted by the cloud will be less than the Planck value; i.e., its emissivity will be less than 1 . This is because the presence of scattering particles gives the cloud a nonzero reflectance, and if there is no transmission, the absorptivity (equal to the emissivity) must be 1 minus the reflectance. In the limit of very large optical depth, $\varepsilon_{b}$ becomes the actual emissivity of the cloud, which we write as $\varepsilon_{\infty, b}$.

Two-stream computations (including SPARTACUS) correctly reduce the vertically upwelling and downwelling longwave fluxes out of top and base of an optically thick cloud from the Planck value, corresponding to an emissivity of less than 1.

For SPARTACUS to capture this effect at cloud sides as well, we need to specify the emissivity $\varepsilon_{\infty, b}$ directly. This quantity may be calculated from the two-stream equations by treating the optically thick cloud as semi-infinite. In this particular case, we neglect horizontal transport terms, because for the thin layers at cloud edges that are relevant to this calculation, the lateral gain and loss terms are dominated by the $\gamma_{1}$ and $\gamma_{2}$ terms. The two-stream equations in this case have been solved by various authors, such as Petty [2006] whose equation 13.45 described the albedo of a semi-infinite cloud, $r_{\infty}$. The emissivity is then

$$
\varepsilon_{\infty, b}=1-r_{\infty}=\frac{2 \sqrt{1-\omega}}{\sqrt{1-\omega g}+\sqrt{1-\omega}},
$$

where $g$ is the asymmetry factor of the medium in region $b$.

\subsubsection{Parameterization Depending on Optical Depth}

For general optical depth, the correct parameterization of the edge flux is more complicated, due to the interaction of several effects that partially compensate each other, and cannot be analytically solved. We find that a satisfactory empirical approximation of $\varepsilon_{b}$ is

$$
\varepsilon_{b}(\tau, \omega, g)=\frac{\varepsilon_{0, b}-\varepsilon_{\infty, b}(\omega, g)}{\tau(1-\omega)+1}+\varepsilon_{\infty, b}(\omega, g) .
$$


This distribution has limiting values that agree with the analytical results for $\tau \rightarrow 0$ (found in section 3.3.1) and $\tau \rightarrow \infty$ (found in section 3.3.2). Note that in terms of practical application of (18) in the SPARTACUS radiation scheme, $\tau$ is the horizontal optical depth of a typical cloud in a given layer, which can be estimated by multiplying the extinction coefficient by the typical cloud diameter. In the implementation of SPARTACUS, we use as a measure of this the similar effective cloud diameter (discussed in section 4) that we also use for characterizing cloud edge length, as outlined by Hogan and Shonk [2013] and Jensen et al. [2008].

To check that treatment of lateral and vertical cloud edge fluxes is consistent, we repeat the SPARTACUS calculations shown in Figure 4 for a homogeneous, isothermal, cubic cloud in vacuum, but this time with the new parameterization for $\varepsilon_{b}$ (shown by the solid lines). This time the ratio $G_{\text {side }} / G_{\text {base }}$ is much closer to the value of 1 expected from theory and found from fully 3-D calculations using MYSTIC and constitutes a significant improvement on the results without the parameterization.

\section{Description of Cloud Geometry in Terms of Effective Cloud Edge Length}

4.1. Theory

In order to determine radiative fluxes correctly, we need to provide SPARTACUS with an appropriate description of cloud geometry. SPARTACUS assumes that the flux between clear and cloudy regions is proportional to the length of the cloud edges in the horizontal plane, with coefficients of proportionality as derived in the previous section. In sections 2 and 3 , it was found that this parameterization works well for idealized clouds with smooth edges, such as cubes. We will see in this section that for realistic clouds with irregular edges finding the appropriate edge length for input in SPARTACUS is less straightforward, as using our parameterization with cloud edge lengths measured in high-resolution cloud fields overestimates the 3-D effect. It appears that the "effective" cloud edge length for radiation is less than the measured cloud edge length, which we hypothesize is due to two effects:

1. Irregular, fractal cloud edges. Realistic cloud edge length is determined by cloud geometry on a range of scales from the overall cloud size down to the distance between cloud particles. In practical scenarios, we only have model or observation data down to a finite grid resolution, which is at best tens of meters, so some structure is missing. Cloud geometry is fractal, meaning that the measured edge length $L$ is sensitive to small-scale features and increases with decreasing grid spacing $\Delta x$ :

$$
L(\Delta x) \propto \Delta x^{1-D},
$$

where $D$ is the fractal dimension of the cloud perimeter, which for a large class of fractals can be found as the exponent that relates the object's perimeter to the square root of the area. Studies of both observed [e.g., Cahalan and Joseph, 1989] and simulated clouds [Siebesma and Jonker, 2000] have found a perimeter fractal dimension of about $4 / 3$ for small shallow cumulus. Thus, we have a fundamental observational problem: Cloud edge length is not a well-defined quantity but depends on resolution.

On the other hand, due to the 3-D transport effects that we are including, the radiation fields are much smoother than the cloud water fields, an effect known as radiative smoothing. Marshak et al. [1995] found a fractal dimension of 1.14 for the albedo field (which should be very similar to the flux field), and hypothesized that it might be even closer to 1 but for numerical imprecisions. In particular, small-scale concave features of the cloud edge are smoothed out in flux fields. This is appropriate, as most of the radiative flux escaping from these parts of the cloud edge will reenter neighboring sections of the cloud instead of contributing to clear-sky fluxes. Hence, the effective cloud edge length we are seeking should not include the added length contributed by these small concave features, but should instead follow the edge of an approximation to the cloud that is smooth and convex up to the same scale that the flux field is. Radiative smoothing is most pronounced in the shortwave spectral region, where multiple scattering leads to isotropic diffuse fluxes, but the effect is also relevant for isotropic emission in the longwave. There is also nonnegligible scattering in the longwave (for the droplet size and wavelength considered here, the single scattering albedo $\omega$ is 0.6 ), which we include in our model. This explains why the assumption of flux $\propto L$ works quite well for idealized Euclidean clouds such as cubes with $D=1$, but for realistic clouds at high resolution the measured edge length is higher than the radiative effective edge length of the flux field, and hence using the measured edge length in the radiative transport parameterization leads to an overestimation of the 3-D transport.

2. Cloud clustering. Another effect that can reduce the 3-D transport in cloud fields with multiple clouds is that radiation leaving the side of one cloud can be intercepted by another cloud. SPARTACUS allows this to 
(a)

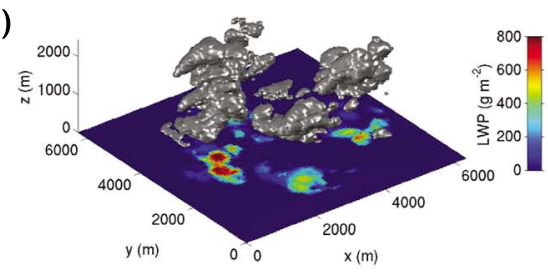

(c)

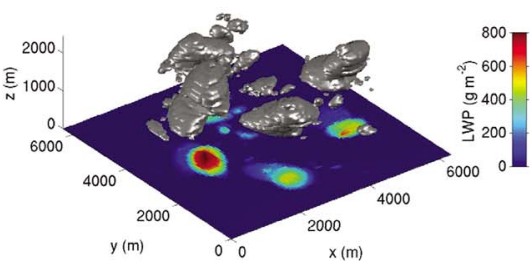

(b)

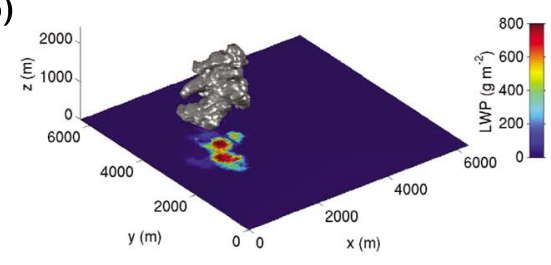

(d)

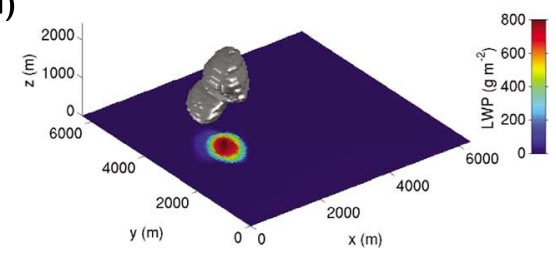

Figure 5. Cloud fields of the four experiments described in section 4.2: (a) cumulus cloud field, (b) isolated cumulus cloud, (c) ellipsified cloud field, and (d) isolated ellipsified cloud. The gray shapes are 3-D contours of cloud liquid water content larger than $0.001 \mathrm{~g} \mathrm{~m}^{-3}$, while the color field shown below is the liquid water path of each vertical column. The domain measures $6.4 \mathrm{~km}$ by $6.4 \mathrm{~km}$, grid resolution is $67 \mathrm{~m}$ in the horizontal and $40 \mathrm{~m}$ in the vertical, and a realistic temperature profile based on a radiosonde sounding is used [Hinkelman et al., 2005].

happen but assumes that the clouds are randomly spaced throughout the domain. In realistic cloud fields, groups of clouds may tend to cluster together, making the chance of radiation from one cloud's sides being intercepted by other clouds higher than if they were randomly spaced. Underestimating this reduction of 3-D transport in SPARTACUS would add to the overestimation of 3-D effects.

\subsection{Experiments}

In order to separate, quantify, and account for these effects, we have designed a set of experiments based on a realistic large-eddy simulation (LES) of a continental shallow cumulus cloud field by Hinkelman et al. [2005] used in the Intercomparison of 3-D Radiation Codes (I3RC) project [Cahalan et al., 2005]. Clouds are described by their liquid water content and effective cloud particle radius in a periodic domain measuring $6.4 \mathrm{~km}$ in each horizontal dimension with a resolution of $67 \mathrm{~m}$ in the horizontal and $40 \mathrm{~m}$ in the vertical. The temperature profile is taken from a radiosonde sounding at the Atmospheric Radiation Measurement (ARM) program's Southern Great Plains site in Oklahoma, USA [Hinkelman et al., 2005]. To isolate cloud effects, we do not include atmospheric gases, aerosol, surface emission, or reflection.

We start with the full cumulus cloud field (depicted in Figure 5a), centered within the domain. In order to clearly isolate differences due to treatment of 3-D effects, we remove cloud internal inhomogeneity effects by assuming constant effective droplet radius throughout, and constant averaged liquid water content within each layer, making the 3-D calculations directly comparable to SPARTACUS with two regions, clear and cloudy. We label the pixels belonging to each individual cloud in the cloud field using an algorithm based on the object labeling code of Stein et al. [2014]. This algorithm considers two pixels to be part of the same object if they share an edge face but not if they just share a corner. In Experiments a and c, we consider the full cloud field containing 55 clouds, showing the cloud clustering effect. For Experiments b and d, we select one isolated cumulus cloud and remove all others. Without neighboring clouds there is no cloud clustering effect, allowing us to observe the irregular edge effect alone.

We estimate the irregular edge effect by using two different cloud edge lengths as input for SPARTACUS: In Experiment $a$ and $b$, we calculate the cloud edge length from high-resolution cloud contours, found by reducing the cloud field at each height to a 2-D field of ones (cloud present) and zeros (no cloud present) and computing the length of the 0.5 contour. As these contours include cloud edge structure at scales likely too small to impact the radiation field, we expect an overestimation of 3-D fluxes in SPARTACUS with this input, the irregular edge effect discussed in section 4.1 (and in Experiment a also overestimation due to cloud clustering).

For Experiments $c$ and $d$, we calculate the edge lengths by fitting an ellipse to each horizontal cross section of each cloud of Experiments $a$ and b respectively, using the method of Hogan et al. [2012], which ensures that the fitted ellipse preserves the aspect ratio, orientation, and area as much as possible given the finite pixel size. 


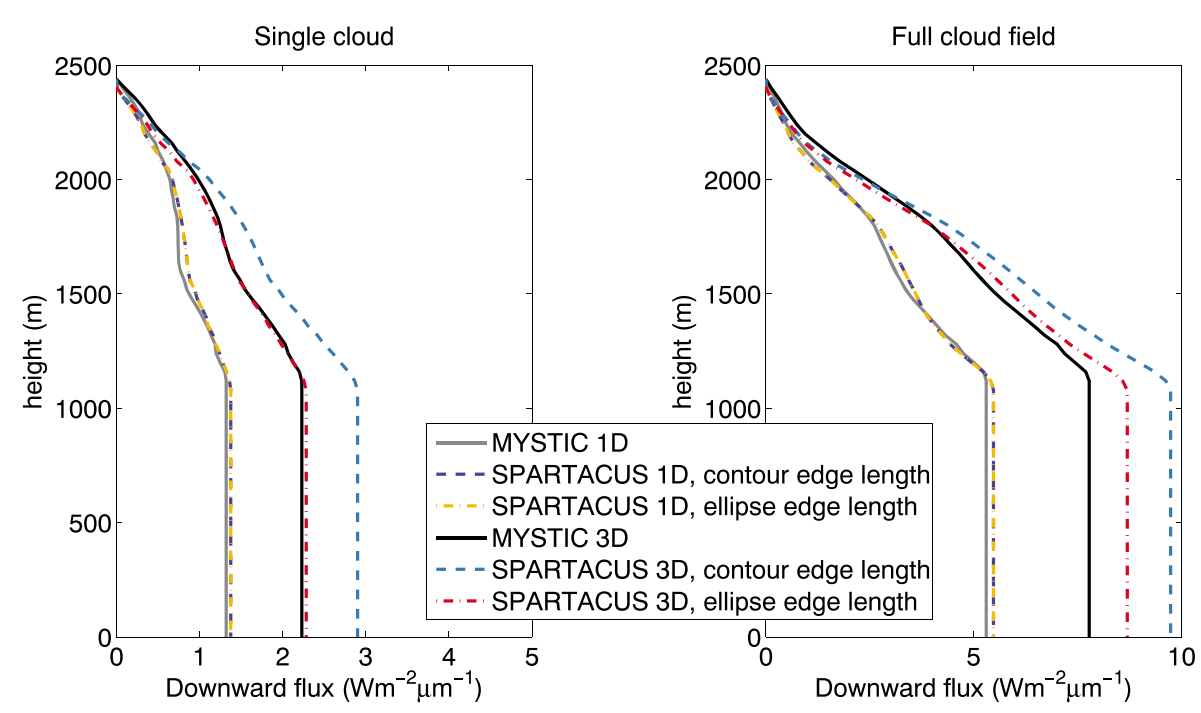

Figure 6. Downward domain-averaged monochromatic fluxes at wavelength $\lambda=10.7 \mu \mathrm{m}$, from MYSTIC and from SPARTACUS using contour edge length and ellipsified edge length. (left) Profiles for the single-cloud case and (right) profiles for the whole cloud field. Note that in the 1-D calculations, the cloud edge length does not matter, so the 1-D profiles with contour edge length and ellipse edge length coincide.

The resulting ellipse is a convex smooth approximation of the cloud. The field's ellipse cloud edge length is the sum of the exact ellipse perimeters of all the ellipses in each slice. We refer to this process as "ellipsification." The idea is to estimate the radiative effective cloud edge length for use in SPARTACUS by maintaining the largest-scale properties of cloud geometry but excluding the influence of smaller-scale features irrelevant for radiation. Thus, Experiment $\mathrm{c}$ includes the cloud clustering effect but not the irregular edge effect, while Experiment $d$ excludes both effects. Ellipse cloud edges are shorter than contour cloud edges by a (vertically averaged) factor of 1.9 for the isolated cloud and 1.5 for the cloud field - the reduction is less for the full cloud field because it contains small clouds of only a few grid boxes, which do not show irregular features at the given resolution; therefore, ellipsification has less impact.

For each cloud field, we choose the optimum height-independent cloud overlap parameter for SPARTACUS's cloud overlap scheme [Shonk et al., 2010] so as to give the correct total cloud cover. We compare the results of SPARTACUS, including the edge flux parameterization described in section 3.3.3 and with the cloud edge length inputs discussed above, to fully 3-D MYSTIC calculations for the original cloud field (Experiments a and c) and original isolated cumulus cloud (Experiments b and d).

\subsection{Results for Cloud Geometry Description}

As seen in Figure 6, SPARTACUS's results agree well with MYSTIC in the 1-D case (without horizontal transport). In 3-D, MYSTIC and SPARTACUS with ellipse edge length agree well for the single cloud, while when using contour edge length or in the presence of neighboring clouds, the effects discussed in section 4.1 lead SPARTACUS to overestimate the 3-D flux. To test how representative this particular cloud is, we have run analogous single-cloud calculations for every cloud in the cloud field that is larger than four grid boxes, using SPARTACUS with ellipse edge length and MYSTIC. Figure 7 shows a comparison of the 3-D factor $f_{1 \text { Dcorrection }}=F_{3 \mathrm{D}}^{\downarrow} / F_{1 \mathrm{D}}^{\downarrow}$ at the surface between SPARTACUS and MYSTIC for each single cloud, where the symbols' colors indicate the cloud's total cloud cover. The two codes' results agree reasonably well, especially for the largest clouds in the field (in dark blue colors), which dominate the total cloud field results. The cloud selected for the single-cloud experiment above is typical of these large clouds. For the smallest clouds (in pale cyan), results agree less closely due to numerical noise (the three clouds that show a 3-D factor larger than 3 in MYSTIC are the three smallest clouds in the ensemble). The agreement between MYSTIC and ellipsified SPARTACUS in single-cloud cases confirms that SPARTACUS's overestimation of 3-D effects for the full cumulus field is due to a combination of the two effects suggested in section 4.1 and that our ellipsification method succeeds in removing the error due to small-scale irregular cloud edges. This is important: it suggests that for cumulus clouds, the radiatively effective cloud edge length is well represented by the perimeter of an ellipse fitted to the cloud 


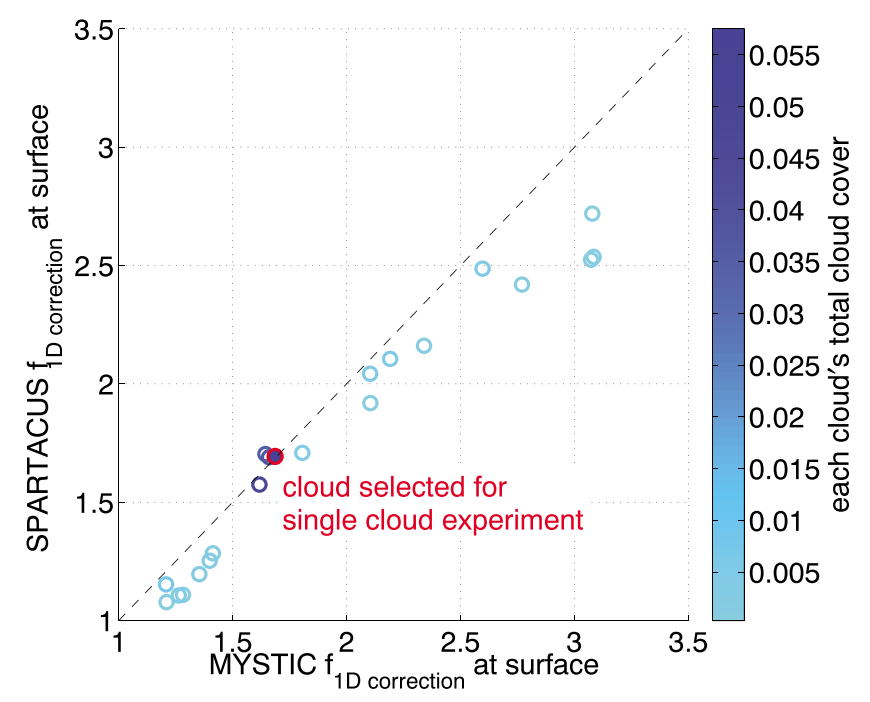

Figure 7. The 3-D factor $f_{1 \text { Dcorrection }}=F_{3 \mathrm{D}}^{\downarrow} / F_{1 \mathrm{D}}^{\downarrow}$ at the surface for the fluxes from each single cloud larger than four grid boxes in the cloud field, using SPARTACUS with ellipse cloud edge length, plotted against the same factor seen in MYSTIC results. The symbols' colors show each cloud's total cloud cover. The filled, red-edged symbol denotes the cloud used in the single-cloud experiment discussed above. The 1-to-1 line is shown dashed in black.

edge at each height. More experiments are needed to confirm if an analogous method would work for other cloud types.

This still leaves the error caused by cloud clustering. To determine how clustered the cloud field is, we calculate the average horizontal distance between centers of mass of each cloud and its nearest neighbor $\overline{d_{\text {next }}}$, allowing for periodic boundary conditions at domain boundaries, as do the radiation calculations. In the full cloud field with 55 clouds, $\overline{d_{\text {next }}}$ is $276.4 \mathrm{~m}$. Distributing the same number of clouds randomly in a domain of the same size results in a mean nearest neighbor distance of $\overline{d_{\text {next,rand }}}=432.7 \mathrm{~m}$ (averaged over $10^{5}$ random distribution realizations to ensure representative sampling). This shows that in reality, our cloud field is significantly more clustered than in SPARTACUS's random distribution assumption, therefore intercepting more of the outgoing flux from cloud sides than SPARTACUS accounts for. We can compensate for this by reducing the cloud edge length and thereby also the cloud side flux. By running SPARTACUS repeatedly with a range of reduction factors, we can determine the optimum reduced edge length resulting in exact agreement between SPARTACUS and MYSTIC in each case. For the isolated cloud, without any cloud clustering to compensate, the optimum input edge length is about the ellipse edge length (at 0.94 times the ellipse edge length), while for the cloud field it is 0.69 times the ellipse edge length. This shows that using the ellipse edge length and multiplying by a factor of 0.69 to correct for clustering is a good estimate for the radiatively effective cloud edge length in this cumulus field. Further work will be needed to see how typical this value is for cumulus clouds and to determine the appropriate reduction factor depending on degree of clustering for any cloud type.

\section{Conclusions}

Three-dimensional radiative effects systematically and significantly increase the longwave cloud radiative effect of cumulus clouds, an effect that has been largely neglected in the literature. Hogan and Shonk [2013] presented an idea for how to rigorously capture 3-D shortwave cloud effects in a two-stream radiation scheme at a numerical cost suitable for use in a global model. This two-part paper provides many improvements to their method, and we refer to the resulting radiation scheme as SPARTACUS.

This first part has developed the longwave capabilities of SPARTACUS. We have first proposed a benchmark case: an isolated, homogeneous, isothermal, cubic cloud in vacuum, for which the 3-D effect is known theoretically. In the optically thick limit, 3-D effects increase its cloud radiative effect by a factor of exactly 3 . This benchmark has been used to ensure consistent treatment of horizontal and vertical fluxes at every step of the method, since the flux out of each of the six faces of the cube should be the same. It is shown that if the diffuse radiation is assumed to be traveling in two discrete directions, then in computing fluxes out of cloud sides, a zenith angle of $\theta_{3-D}=57.52^{\circ}$ must be used, rather than the $\theta_{1}=53^{\circ}$ most often used for vertical transport 
calculations in the longwave. We also find that in the longwave the spatial distribution of fluxes within the clouds is important and develop a parameterization for the flux values at cloud sides that represents the buildup of fluxes toward cloud edge in the optically thin case and cloud edge emissivity in the optically thick case.

The decisive cloud geometry parameter for determining the cloud side fluxes correctly is the effective cloud edge length relevant for radiation. For idealized clouds such as cubes, this is simply the geometric edge length. For realistic high-resolution cloud fields, we find that this length is lower than the measured cloud edge length for two reasons. First, the radiation field is smoother than the high-resolution cloud water field, so essentially the small-scale fluctuations of cloud water are irrelevant for radiation. We find that for cumulus clouds, a good approximation of the radiatively effective cloud edge length at a given height is the perimeter of an ellipse fitted to the cloud boundary such that area and aspect ratio are preserved. Second, clouds tend to be more clustered than would result from the random distribution assumed by SPARTACUS, enhancing the chance of radiation emitted from a cloud side being intercepted by a neighboring cloud and therefore reducing the effective length of cloud sides from which radiation escapes. To determine this reduction correctly, we need to consider how strongly the clouds cluster. We have proposed here a simple empirical adjustment to the effective cloud edge length to approximately account for this effect, but clearly further observational work is required to quantify the extent to which clouds cluster in reality and its impact on 3-D radiative transfer.

Part 2 of this paper [Hogan et al., 2016] describes much of the further work needed to prepare SPARTACUS for use in a large-scale atmospheric model. They introduce an elegant method for solving the two-stream equations modified to include 3-D effects and incorporate full representation of gas absorption and surface effects, enabling them to perform a realistic comparison between SPARTACUS and fully 3-D broadband radiative transfer calculations for cloud fields in both the shortwave and longwave. They discuss practical implementation and numerical cost. However, further analysis of observations and cloud-resolving simulations is required to extract the radiatively effective cloud edge length for different cloud fields and to parameterize it as a function of variables available in a large-scale model. The findings in section 4 of this paper should provide useful guidance on the analysis that would be most useful to provide the information required to SPARTACUS.

Acknowledgments

We thank Susanne Crewell for many valuable discussions and suggestions throughout the course of this work, Jonathan Shonk for providing the Matlab code that was used in the Hogan and Shonk [2013] paper, Thorwald Stein for providing the object labeling code used in section 4, and Christopher Westbrook for suggesting the analytical solution to equation (16). The first author was supported by a doctoral studentship in the ITaRS project from the European Union Seventh Framework Programme, ITN Marie Curie Actions Programme (2012-2016) under grant agreement 289923. The SPARTACUS model and data used in this paper are available on request from the second author, Robin Hogan (please email r.j.hogan@ecmwf.int). The cloud case our analysis is based on is available at http://i3rc.gsfc.nasa.gov/input/ remsensing.html (Case 4).

\section{References}

Benner, T. C., and K. F. Evans (2001), Three-dimensional solar radiative transfer in small tropical cumulus fields derived from high-resolution imagery, J. Geophys. Res., 106, 14,975-14,984.

Boucher, O. D. R., et al. (2013), Clouds and aerosols, in Climate Change 2013: The Physical Science Basis. Contribution of Working Group I to the Fifth Assessment Report of the Intergovernmental Panel on Climate Change, edited by T. Stocker Cambridge Univ. Press, Cambridge, U. K., and New York.

Cahalan, R. F., and J. H. Joseph (1989), Fractal statistics of cloud fields, Mon. Weather Rev., 117(2), $261-272$.

Cahalan, R. F., et al. (2005), The I3RC: Bringing together the most advanced radiative transfer tools for cloudy atmospheres, Bull. Am. Meteorol. Soc., 86, 1275-1293, doi:10.1175/BAMS-86-9-1275.

Cole, J., H. Barker, W. O'Hirok, E. Clothiaux, M. Khairoutdinov, and D. Randall (2005), Atmospheric radiative transfer through global arrays of 2D clouds, Geophys. Res. Lett., 32, L19817, doi:10.1029/2005GL023329.

Di Giuseppe, F., and A. M. Tompkins (2003), Three-dimensional radiative transfer in tropical deep convective clouds, J. Geophys. Res., 108(D23), 4741, doi:10.1029/2003JD003392.

Elsasser, W. (1942), Heat Transfer by Infrared Radiation in the Atmosphere, Harvard Meteorological Studies, vol. 6, Harvard Univ. Press, Milton, Mass.

Emde, C., and B. Mayer (2007), Simulation of solar radiation during a total eclipse: A challenge for radiative transfer, Atmos. Chem. Phys., 7(9), 2259-2270.

Fu, Q., K. Liou, M. Cribb, T. Charlock, and A. Grossman (1997), Multiple scattering parameterization in thermal infrared radiative transfer, J. Atmos. Sci., 54, 2799-2812.

Gounou, A., and R. J. Hogan (2007), A sensitivity study of the effect of horizontal photon transport on the radiative forcing of contrails, J. Atmos. Sci., 64, 1706-1716.

Heidinger, A. K., and S. K. Cox (1996), Finite-cloud effects in longwave radiative transfer, J. Atmos. Sci., 53, 953-963.

Hinkelman, L. M., B. Stevens, and K. F. Evans (2005), A large-eddy simulation study of anisotropy in fair-weather cumulus cloud fields, J. Atmos. Sci., 62(7), 2155-2171.

Hogan, R. J., and J. K. P. Shonk (2013), Incorporating the effects of 3D radiative transfer in the presence of clouds into two-stream multilayer radiation schemes, J. Atmos. Sci., 70, 708-724.

Hogan, R. J., L. Tian, P. R. Brown, C. D. Westbrook, A. J. Heymsfield, and J. D. Eastment (2012), Radar scattering from ice aggregates using the horizontally aligned oblate spheroid approximation, J. Appl. Meteorol., 51, 655-671.

Hogan, R. J., S. A. K. Schäfer, C. Klinger, J. C. Chiu, and B. Mayer (2016), Representing 3-D cloud radiation effects in two-stream schemes: 2. Matrix formulation and broadband evaluation, J. Geophys. Res. Atmos., 121, 8583-8599, doi:10.1002/2016JD024875.

Jakub, F., and B. Mayer (2015), A three-dimensional parallel radiative transfer model for atmospheric heating rates for use in cloud resolving models - The tenstream solver, J. Quant. Spectros. Radiat. Transfer, 163, 63-71.

Jensen, M. P., A. M. Vogelmann, W. D. Collins, G. J. Zhang, and E. P. Luke (2008), Investigation of regional and seasonal variations in marine boundary layer cloud properties from MODIS observations, J. Clim., 21, 4955-4973, doi:10.1175/2008JCLI1974.1. 
Killen, R. M., and R. G. Ellingson (1994), The effect of shape and spatial distribution of cumulus clouds on longwave irradiance, J. Atmos. Sci, 51(14), 2123-2136.

Klinger, C., and B. Mayer (2014), Three-dimensional Monte Carlo calculation of atmospheric thermal heating rates, J. Quant. Spectros. Radiat. Transfer, 144, 123-136.

Klinger, C., and B. Mayer (2016), The Neighboring Column Approximation (NCA) - A fast approach for the calculation of 3D thermal heating rates in cloud resolving models, J. Quant. Spectros. Radiat. Transfer, 168, 17-28.

Marshak, A., A. Davis, W. Wiscombe, and R. Cahalan (1995), Radiative smoothing in fractal clouds, J. Geophys. Res., 100, 26,247-26,261.

Mayer, B., S. Hoch, and C. Whiteman (2010), Validating the MYSTIC three-dimensional radiative transfer model with observations from the complex topography of Arizona's meteor crater, Atmos. Chem. Phys., 10(18), 8685-8696.

Mayer, B. (2009), Radiative transfer in the cloudy atmosphere, in ERCA 2008-From the Human Dimensions of Global Environmental Change to the Observation of the Earth From Space, vol. 1, edited by C. Boutron, pp. 75-99, EPJ Web of Conf., Grenoble, France, doi:10.1140/epjconf/e2009-00912-1.

Petty, G. W. (2006), A First Course in Atmospheric Radiation, Sundog Publ., 400-402 pp., Wisconsin.

Pincus, R., and K. F. Evans (2009), Computational cost and accuracy in calculating three-dimensional radiative transfer: Results for new implementations of Monte Carlo and SHDOM, J, Atmos. Sci., 66, 3131-3146, doi:10.1175/2009JAS3137.1.

Pincus, R., H. W. Barker, and J.-J. Morcrette (2003), A fast, flexible, approximate technique for computing radiative transfer in inhomogeneous cloud fields, J. Geophys. Res., 108, 4376, doi:10.1029/2002JD003322.

Shonk, J. K., and R. J. Hogan (2008), Tripleclouds: An efficient method for representing horizontal cloud inhomogeneity in 1D radiation schemes by using three regions at each height, J. Clim., 21, 2352-2370.

Shonk, J. K. P., and R. J. Hogan (2010), Effect of improving representation of horizontal and vertical cloud structure on the Earth's global radiation budget. Part II: The global effects, Q. J. R. Meteorol. Soc., 136, 1205-1215.

Shonk, J. K. P., R. J. Hogan, J. M. Edwards, and G. G. Mace (2010), Effect of improving representation of horizontal and vertical cloud structure on the Earth's global radiation budget. Part I: Review and parametrization, Q. J. R. Meteorol. Soc., 136, 1191-1204.

Siebesma, A., and H. Jonker (2000), Anomalous scaling of cumulus cloud boundaries, Phys. Rev. Let., 21, 214-217.

Stein, T. H., R. J. Hogan, K. E. Hanley, J. C. Nicol, H. W. Lean, R. S. Plant, P. A. Clark, and C. A. Halliwell (2014), The three-dimensional morphology of simulated and observed convective storms over southern England, Mon. Weather Rev., 142, 3264-3283.

Takara, E., and R. Ellingson (2000), Broken cloud field longwave-scattering effects, J. Atmos. Sci., 57(9), 1298-1310.

Zhong, W., R. J. Hogan, and J. D. Haigh (2008), Three-dimensional radiative transfer in midlatitude cirrus clouds, Q. J. R. Meteorol. Soc., 134, $199-215$.

Zuidema, P., and K. F. Evans (1998), On the validity of the independent pixel approximation for boundary layer clouds observed during ASTEX, J. Geophys. Res., 103, 6059-6074. 